\title{
MicroRNA-192 inhibits cell proliferation and induces apoptosis in human breast cancer by targeting caveolin 1
}

\author{
PINJIA CHEN $^{1 *}$, YUN FENG $^{2 *}$, HAIGE ZHANG $^{1}$, XINPENG SHI $^{1}$, BIN LI $^{1}$, WENCUI JU ${ }^{1}$, \\ XIURONG YU ${ }^{1}$, NAN ZHANG ${ }^{1}$ and XIAOYONG LUO ${ }^{1}$ \\ ${ }^{1}$ Department of Oncology and ${ }^{2}$ Surgical Department, \\ The Affiliated Luoyang Central Hospital of Zhengzhou University, Luoyang, Henan 471000, P.R. China
}

Received August 12, 2018; Accepted January 25, 2019

DOI: $10.3892 /$ or.2019.7298

\begin{abstract}
It has been demonstrated that microRNA-192 (miR-192) serves important roles in different cancer types, including breast cancer, prostate cancer and colorectal cancer. However, its biological role and function in breast cancer remains largely unknown. The present study aimed to determine the role of miR-192 in breast cancer. In the present study, one normal breast and two breast tumor cells lines were used, which included the normal mammary fibroblast cell line Hs578Bst, a more aggressive breast tumor cell line MDA-MB-231 and a less aggressive breast tumor cell line MCF-7. The effect of miR-192 on proliferation of breast cancer cells was detected with an MTT assay. Western blot analysis was performed to determine protein expression of caveolin 1 (CAV1). A lentiviral vector that overexpresses pre-miR-192 and control lentiviral packaging plasmids were used in the present study. The Student's t-test was performed to analyze the significance of differences between samples. In the present study, it was determined that the expression of miR-192 is downregulated in breast cancer, compared with the adjacent normal tissues. Overexpression of miR-192 significantly inhibited cell proliferation, and induced cell apoptosis and cell cycle arrest in MCF7 and MDA-MB-231 cells. Using a bioinformatics method, CAV1 was considered a potential target of miR-192. Furthermore, it was demonstrated that CAV1 is a direct target of miR-192 and its protein expression is negatively regulated by miR-192. Therefore, the present study demonstrated that miR-192 serves an important role as a regulator in breast cancer and the miR-192/CAV1 axis has a potential as a therapeutic target for treatment of breast cancer.
\end{abstract}

Correspondence to: Dr Xiaoyong Luo, Department of Oncology, The Affiliated Luoyang Central Hospital of Zhengzhou University, 288 Zhongzhou Road, Luoyang, Henan 471000, P.R. China E-mail: xiaoyongluo68@aliyun.com

*Contributed equally

Key words: microRNA, microRNA-192, caveolin 1, breast cancer, cell proliferation, apoptosis, cell cycle

\section{Introduction}

Breast cancer is one of the most frequently diagnosed malignancy in females with an estimated 1.5 million new cases worldwide each year (1). Due to the fast development of diagnosis and novel treatment techniques, the incidence has been decreasing in the developed and developing world (2). However, it remains the second leading cause of mortality from cancer in women (3). Thus, investigating a novel diagnosis method and determining a novel treatment target remains urgent. A previous study was conducted to identify novel markers that control and regulate cell growth and differentiation, which are associated with tumor growth, formation and progression of breast cancer (3).

MicroRNAs (miRNAs/miRs) are small non-coding single-stranded RNAs, which contain 20-25 nucleotides, that serve important roles in RNA silencing and post-transcriptional regulation of gene expression in multicellular organisms (4). Animal miRNAs are generally complementary to a site in the 3'untranslated region (UTR) of the target mRNA by perfect or near perfect base paring to promote the cleavage of the target RNA, thus inducing degradation of the target gene (5). miRNAs are considered to serve roles in cancer development, progression and metastasis, and during the cancer development, numerous miRNAs, including miR-15, miR-16, miR-21 and miR-29a have been discovered. Additionally, miRNAs can serve oncogenic or tumor suppressive roles in carcinogenesis through negative regulation of target gene expression (6-8).

In breast cancer, various miRNAs have been discovered and they have been demonstrated to be deleted, downregulated or upregulated, such as the miR-17-92 cluster, which is upregulated, and miR-143 and miR-145, which are deleted (9). They also have been determined to have oncogenic or tumor suppressive effects, and serve important roles in tumor initiation, antitumor drug resistance and advanced tumor metastasis (10-13). Among the miRNAs, miR-192 was confirmed by Lim et al (14) in 2003. miR-192 is considered to be positive regulator of $\mathrm{p} 53$, which is a human tumor suppressor gene (15). miR-192 is also reported to be overexpressed in gastric cancer, hepatocellular carcinoma and neuroblastoma, while downregulated in colorectal cancer and hematological disorders, as well as in lymphoblastic leukemia (16-20). However, its role in breast cancer development and formation remains unknown. 
In the present study, the results indicated that the miR-192 was significantly decreased in the tumor tissue, compared with adjacent normal tissue. Upregulation of miR-192 inhibits tumor cell proliferation by inducing of the tumor cell apoptosis cell cycle arrest. Notably, using a bioinformatics method, it was demonstrated that caveolin 1 (CAV1) is a direct target of miR-192 and its protein expression is negatively regulated by miR-192. Therefore, these results demonstrated that miR-192 serves an important role in the regulation of breast tumor cell proliferation and apoptosis, and the miR-192/CAV1 axis may have a potential as a therapeutic target for treatment of breast cancer.

\section{Materials and methods}

Patient samples. A total of 58 specimens from women with breast cancer and adjacent normal tissues samples were collected from The Affiliated Luoyang Central Hospital of Zhengzhou University (Luoyang, China) from January 2015 to March 2017. The patients had a mean age of $56 \pm 12$ years, and did not receive radiotherapy, chemotherapy or any other treatment prior to or following the operation. Patient characteristics are listed in Table SI. Tumor surgical specimens, tumor lumps and tumor adjacent normal tissues that were at least $2 \mathrm{~cm}$ from the edge of the tumor were collected, snap-frozen in liquid nitrogen and stored at $-80^{\circ} \mathrm{C}$ for miR-192 and CAV1-associated assays. Written informed consent was obtained from all the study participants. The use of tissue samples was approved by the Ethics Committee of the Affiliated Luoyang Central Hospital of Zhengzhou University.

Cell culture and transfection. A total of 3 breast and breast tumor cells lines were used in the present study, which includes the normal mammary fibroblast cell line Hs578Bst, a more aggressive breast tumor cell line MDA-MB-231 and a less aggressive breast tumor cell line MCF-7. All these cell lines were obtained from American Type Culture Collection (Manassas, VA, USA) and maintained in RPMI-1640 (Gibco; Thermo Fisher Scientific, Inc., Waltham, MA, USA) with $10 \%$ fetal bovine serum (FBS; Gibco; Invitrogen) and $1 \%$ antibiotics (Gibco; Thermo Fisher Scientific, Inc.) in an atmosphere of humidified air containing 5\% $\mathrm{CO}_{2}$. MCF-7 and MDA-MB-231 cells were transfected with miR-192 mimics (miR-192 mimics: 5'-CUGACCUAUGAAUUGACA GCC-3') or miR-Control (5'-UUCUCCGAACGUGUCACG UTT-3') (Shanghai Genepharma Co., Ltd., Shanghai, China) at $10 \mathrm{pmol} / 1 \times 10^{3}$ cells using Lipofectamine ${ }^{\circledR} 2000$ (Invitrogen; Thermo Fisher Scientific, Inc.) according to the manufacturer's protocols.

Detection of cell proliferation with an MTT assay. The effect of miR-192 on proliferation of breast cancer cells was detected with an MTT assay. Briefly, MCF-7 and MDA-MB-231 cells were plated in 96 -well plates $\left(3 \times 10^{3} /\right.$ well $)$. After incubation for $24 \mathrm{~h}$ in a $37^{\circ} \mathrm{C}$ incubator with $5 \% \mathrm{CO}_{2}$, the cells were transfected with miR-192 mimics (30 pmol) or miR-Control (30 pmol) for 12, 24 and $48 \mathrm{~h}$ using Lipofectamine ${ }^{\circledR} 2000$. Subsequently, the MTT solution $(0.5 \mathrm{mg} / \mathrm{ml}$; Sigma-Aldrich; Merck KGaA, Darmstadt, Germany) was added to each well $\left(20 \mu \mathrm{l} /\right.$ well). After an additional $4 \mathrm{~h}$ incubation at $37^{\circ} \mathrm{C}$,
MTT solution was discarded and $200 \mathrm{ml}$ dimethyl sulfoxide (Sigma-Aldrich; Merck KGaA) was added, and the plates were shaken gently. The absorbance was measured on an ELISA reader at a wavelength of $570 \mathrm{~nm}$. For the colony formation assay, cells were counted and plated in 12-well plates (in triplicate) at 100 cells/well. Fresh complete RPMI-1640 medium was replaced every 3 days. The number of viable colonies was determined after 14 days culture at $37^{\circ} \mathrm{C}$, and the colonies were fixed with $100 \%$ methanol for $10 \mathrm{~min}$ at room temperature and stained with $0.5 \mathrm{~g}$ crystal violet $(0.05 \% \mathrm{w} / \mathrm{v})$ for $15 \mathrm{~min}$ at room temperature. Images of the colonies were captured using a digital camera (Singer Instruments) and colonies were counted. Each experiment was performed in triplicate and repeated for at least three times.

Flow cytometry detection of cell cycle and apoptosis. MCF-7 and MDA-MB-231 cells transfected with miR-192 mimics and miR-Control were plated in 6-well plates in complete RPMI-1640 medium at $1 \times 10^{5}$ cells/well (Gibco; Thermo Fisher Scientific, Inc.) for $24 \mathrm{~h}$ at $37^{\circ} \mathrm{C}$. Subsequently, the cells were cultured with FBS-free RPMI-1640 medium for 48 h; the medium was then replaced with complete RPMI-1640 medium for another $24 \mathrm{~h}$ at $37^{\circ} \mathrm{C}$. Cells were then collected by centrifugation at $500 \mathrm{x} \mathrm{g}$ for $10 \mathrm{~min}$ at room temperature, fixed in $95 \%$ ethanol for $20 \mathrm{~min}$ at room temperature, incubated at $-20^{\circ} \mathrm{C}$ overnight and then washed twice with PBS. The cells were resuspended in $1 \mathrm{ml}$ FACS solution with propidium iodide (PI; PBS, 0.1\% Triton X-100, $60 \mu \mathrm{g} / \mathrm{ml} \mathrm{PI,} 0.1 \mathrm{mg} / \mathrm{ml}$ DNase-free RNase and $0.1 \%$ trisodium citrate) and incubated on ice for $30 \mathrm{~min}$. Cells were analyzed using a FACSCalibur flow cytometer (BD Biosciences, San Jose, CA, USA). A total of $1 \times 10^{4}$ cells were gated and counted for each sample.

To identify apoptotic cells ratio, Annexin V and PI double staining was performed using an Annexin V-Fluorescein Isothiocyanate (FITC) Apoptosis Detection kit (Becton-Dickinson and Company, Franklin Lakes, NJ, USA), according to the manufacturer's protocols. After the MCF-7 and MDA-MB-231 cells were transfected with miR-192 mimics/miR-Control for $48 \mathrm{~h}, 5 \times 10^{5}$ cells were collected by centrifugation at $1,000 \mathrm{x} \mathrm{g}$ at room temperature for $5 \mathrm{~min}$. Cells were re-suspended in $200 \mu \mathrm{l}$ binding buffer (BD Biosciences), and stained with $5 \mu \mathrm{l}$ FITC Annexin V and $1 \mu \mathrm{l}$ PI solution for $30 \mathrm{~min}$ at room temperature. Cell apoptosis was detected by using a FACSCalibur flow cytometer (BD Biosciences). Apoptotic cells were defined as Annexin V-positive/PI-negative.

Western blotting. Western blot analysis was performed to determine protein expression of CAV1. Cell lysates were prepared by using NP-40 cell lysis buffer (Beyotime Institute of Biotechnology, Haimen, China) and the protein concentration in the supernatants was determined using Bradford protein dye reagent (Bio-Rad Laboratories, Inc., Hercules, CA, USA). A total of $30 \mathrm{mg}$ proteins were resolved by $10 \%$ SDS-PAGE and transferred onto polyvinylidene difluoride membrane. The membranes were blocked with 5\% fat-free dry milk in PBS for $30 \mathrm{~min}$ at room temperature and then incubated with primary anti-CAV1 (1:1,000, cat. no. ab2910; Abcam, Cambridge, MA, USA) and anti-GAPDH (1:1,000, cat. no. ab9485; Abcam) antibodies at $4^{\circ} \mathrm{C}$ overnight. Subsequently, they were incubated with horseradish peroxidase (HRP)-conjugated secondary 
antibodies [goat anti-rabbit IgG H\&L (HRP); 1:3,000, cat. no. ab6721; Abcam] for $2 \mathrm{~h}$ at room temperature. CAV1 and GAPDH proteins were visualized with enhanced chemiluminescence detection reagent (Pierce Biotechnology; Thermo Fisher Scientific, Inc.).

Reverse transcription-quantitative polymerase chain reaction $(R T-q P C R)$. Total RNAs were extracted from the frozen tissue using TRIzol ${ }^{\circledR}$ reagent (Invitrogen; Thermo Fisher Scientific, Inc.) and then total RNA $(0.5 \mu \mathrm{g})$ from each sample was used for cDNA synthesis using the M-MLV Reverse Transcriptase kit (Promega Corporation, Madison, WI, USA) according to the manufacturer's protocol. The specific products of human miR-192 and CAV1 were amplified by qPCR using the following primers: miR-192, 5'-GCTGTTATCTGGGGCGAG GG-3' (forward) and 5'-GGTGGGACCATGAGTGCTGC-3' (reverse); and CAV1, 5'-TGGTTTTACCGCTTGCTGTCT G-3' (forward) and 5'-GCAAGTTGATGCGGACATTGCT' (reverse). Verification of gene expression levels was performed by RT-qPCR using EvaGreen (Biotium, Inc., Freemont, CA, USA). The following thermocycling conditions were used: Initial denaturation at $95^{\circ} \mathrm{C}$ for $5 \mathrm{~min}$; followed by 40 cycles of denaturation at $95^{\circ} \mathrm{C}$ for $10 \mathrm{sec}$, annealing at $60^{\circ} \mathrm{C}$ for $10 \mathrm{sec}$ and extension at $72^{\circ} \mathrm{C}$ for $20 \mathrm{sec}$. U6 and GAPDH were used as the internal control; the primer sequences were as follows: U6, 5'-CGAGCACAGAATCGCTTCA-3' (forward) and 5'-CTC GCTTCGGCAGCACATAT-3' (reverse); and GAPDH, 5'-GAA GGTGAAGGTCGGAGTC-3' (forward) and 5'-GAAGATGGT GATGGGATTTC-3' (reverse). The relative expression levels of miR-192 and CAV1 were calculated with the $2^{-\Delta \Delta C q}$ method (21).

Construction of 3'UTR reporter plasmid and luciferase assay. The putative target genes of miR-192 were predicted by TargetScan (www.targetscan.org),PicTar(pictar.mdc-berlin.de) and miRanda (www.microrna.org/microrna/home.do). The human CAV1 3'UTR harboring miR-192 target sequence and the seed-sequence-mutation (miR-192-3'UTR-mut) were synthesized by Shanghai Genepharma Co., Ltd. (Shanghai, China). The CAV1 3'UTR reporter was generated by inserting the entire 3'UTR or 3'UTR-mut of human CAV1 mRNA into Xhol/NotI sites of psiCHECK-2 vector (Promega Corporation) downstream of the Renilla luciferase gene. For the luciferase assay, $1 \times 10^{5}$ cells were transfected with the CAV1 3'UTR reporter and the miR-192 mimics in a 24-well plate using Lipofectamine ${ }^{\circledR} 2000$, according to the manufacturer's protocols. After $24 \mathrm{~h}$, the firefly and Renilla luciferase activities were measured and analyzed with a Dual Luciferase Reporter Assay kit (Promega Corporation). Relative luciferase activity was estimated by normalizing firefly luciferase activity to that of Renilla for each assay. At $24 \mathrm{~h}$ post-transfection, relative luciferase activity was calculated by normalizing firefly luminescence to Renilla luminescence using the Dual Luciferase Reporter Assay (Promega Corporation) according to the manufacturer's protocol.

Knockdown of CAV1 by small interfering RNA (siRNA). The transient transfection of CAV1 siRNA was performed by using Lipofectamine ${ }^{\circledR} 2000$, according to the manufacturer's protocols. The sequences of siRNAs were as follows: siRNA-CAV1, 5'-AGACGAGCUGAGCGAGAAGCA-3';
siRNA-control, 5'-ACTACCGTTGTTATAGGTG-3', and they were used at a final concentration of $50 \mathrm{nM}$. After transfection for $48 \mathrm{~h}$, the cells were collected, cell lysates were prepared and western blot analysis was performed to analyze the effects of the knockdown. The CAV1 siRNA was purchased from Santa Cruz Biotechnology, Inc. (Dallas, TX, USA).

Tumorigenicity in vivo. The lentiviral vector that overexpresses pre-miR-192 and the control lentiviral packaging plasmid was obtained from Shanghai GeneChem Co., Ltd. (Shanghai, China). A total of 16 nude mice (male BALB/c nude mice; age, 4 weeks; Beijing Vital River Laboratory Animal Technology Co., Ltd., Beijing, Chin) were randomly divided into two groups: The lentiviral control group and lentiviral pre-miR-192 group. Mice were housed in isolated cages under a 12-h light/dark cycle with free access to food and water at $24 \pm 2^{\circ} \mathrm{C}$ and $55 \pm 10 \%$ humidity. MCF-7 cells stably transfected with pre-miR-192 mimics and pre-miR-control were inoculated bilaterally and subcutaneously into the right flanks of nude mice. Tumor growth was monitored and tumor size was measured using vernier calipers every seven days, and the mice were euthanized after four weeks. The volume of the implanted tumor was calculated using the formula: Volume $=\left(\right.$ width $^{2} \times$ length $) / 2$. All animal experiments in the present study were approved by The Ethics Committee of The Affiliated Luoyang Central Hospital of Zhengzhou University.

Statistical analysis. GraphPad Prism software, version 5.0 (GraphPad Software, Inc., La Jolla, CA, USA) was used to analyze data. All the data were obtained from three independent experiments. Data are presented as mean \pm standard error. The Student's t-test was performed to analyze the significance of differences between the samples. One-way ANOVA was carried out for multiple comparisons with Bonferroni's post-hoc test. $\mathrm{P}<0.05$ was considered to indicate a statistically significant difference.

\section{Results}

The expression of miR-192 is decreased in breast cancer tissue and breast cancer cell lines. Total RNAs were extracted from frozen tumor tissue of patients with breast cancer. RT-qPCR was performed to analyze the miR-192 expression level. The relative expression of miR-192 was significantly decreased in the breast cancer tissue, compared with adjacent normal tissues (Fig. 1A). The expression of miR-192 was also evaluated in breast tumor cell lines MCF-7 and MDA-MB-231, as well as the normal breast fibroblast cell line Hs578Bst. RT-qPCR analysis demonstrated that the expression of miR-192 is significantly decreased in breast tumor cell line MCF-7 and MDA-MB-231, compared with normal cell line Hs578Bst (Fig. 1B). Furthermore, the decreased expression of miR-192 was also confirmed in three other breast cancer cell lines, including Hs578T, BCap37 and SK-BR-3 (Fig. S1A). These results indicated that miR-192 expression is significantly reduced in breast tumor tissue and tumor cell lines, which is accordant with previous studies $(16,18)$.

miR-192 inhibits breast tumor cell proliferation. To determine the role of miR-192 in breast tumor cell proliferation, 
breast tumor cells were transfected with miR-192 for 12, 24 and $48 \mathrm{~h}$ and then an MTT assay was performed to determine the cell proliferation. Total RNAs were extracted from all the transfected cells and RT-qPCR was performed to check the overexpression efficiency of miR-192. miR-192 expression was significantly increased following transfection in MCF-7 and MDA-MB-231 cells (Fig. 2A) compared with cells without miR-192 transfection. The MTT assay results indicated that cell proliferation is significantly inhibited after 24 and $48 \mathrm{~h}$ of transfection with miR-192 in MCF-7 and MDA-MB-231 cells (Fig. 2B and C) compared with cells without miR-192 transfection. Colony formation results also demonstrated that cell proliferation is significantly inhibited by the overexpression of miR-192 in MCF-7 and MDA-MB-231 cells (Fig. 2D and E) compared with cells without miR-192 transfection.

MCF-7 stable cell line with overexpression of miR-192 and miR-Control was inoculated into the flank of the mice and then tumor growth was monitored and measured every 7 days. Tumor growth was significantly inhibited by miR-192 expression and the tumor size was smaller in the miR-192 overexpression group, compared with the miR-control group (Fig. 2F and G). These data indicated that miR-192 serves an important role in the regulation of tumor cell proliferation and tumor growth. Therefore, overexpression of miR-192 inhibits tumor cell growth in vivo and in vitro.

Overexpression of miR-192 induces cell apoptosis and cell cycle arrest. To detect the effect of miR-192 on cell apoptotic regulation, cell cycle analysis was performed by staining transfected MCF-7 cells and MDA-MB-231 cells with Annexin-V and PI. Flow cytometry data demonstrated that overexpression of miR-192 significantly increases tumor cell apoptosis with an apoptotic cell ratio of 22.6 and $24.28 \%$ in MCF-7 and MDA-MB-231 cells, respectively, while the apoptotic ratio in the control cells was only 11.67 and $13.74 \%$, respectively (Fig. 3A and B).

The effect of miR-192 on cell cycle was determined by staining cells with PI and analyzed by flow cytometry. The results demonstrated that overexpression of miR-192 induced an increase in the G0/G1 phase $(72.03 \%$ for MCF-7 cells with miR-192 transfection vs. $42.45 \%$ for MCF-7 cells without miR-192 transfection; and $70.38 \%$ for MDA-MB-231 cells with miR-192 transfection vs. $43.83 \%$ for MDA-MB-231 cells without miR-192 transfection) and a decrease in the $\mathrm{S}$ phase in MCF-7 cells (20.11\% for cells without miR-192 transfection vs. $47.64 \%$ for cells with miR-192 transfection), while there was no significant change in the G2 phase (Fig. 3C-F). These results indicated that overexpression of miR-192 significantly increased the number of cells in the G0/G1 phase, while it decreased the number of cells in the $S$ phase in miR-192-transfected MCF-7 and MDA-MB-231 cells.

CAVI negatively regulates the miR-192 expression. Since miRs regulate cellular processes through their target gene (10-13), the target gene for miR-192 was investigated. Bioinformatics analysis predicted that miR-192 may target CAV1 (Fig. 4A). To determine the association between miR-192 and CAV1, PGL3 luciferase reporter vectors were constructed with wild-type or its relevant mutant 3'UTR. Subsequently, 293T cells were co-transfected with miR-192 mimics and the reporter vectors containing wild-type or mutant of CAV1 3'UTR. A luciferase assay was performed to confirm the luciferase intensity of PGL3/Luciferase-CAV1-3'UTR reporter. The results demonstrated that luciferase activity was significantly decreased in the miR-192 and wild-type reporter co-transfected cells (Fig. 4B). Subsequently, the CAV1 RNA expression level was evaluated in miR-192-transfected MCF7 and MDA-MB-231 cells. The RT-qPCR results demonstrated that the CAV1 RNA expression was significantly decreased in miR-192 overexpressed cells (Fig. 4C) compared with in untransfected cells. The correlation between the expression levels of miR-192 and CAV1 was analyzed and the results demonstrated that miR-192 was negatively correlated with CAV1 mRNA expression (Fig. S1B). Furthermore, western blot analysis results further confirmed the decreased expression of CAV1 protein level (Fig. 4D), with a 50\% decrease in miR-192-transfected breast tumor cells (Fig. 4E) compared with in untransfected cells. Collectively, these results indicated that CAV1 expression is negatively associated with miR-192, and CAV1 may be a direct target gene of miR-192.

CAV1 expression increases in the breast tumor tissue. Subsequently, the CAV1 expression in breast tumor tissue and adjacent normal tissues was determined. The RT-qPCR results demonstrated that CAV1 RNA expression is significantly increased in tumor tissue (Fig. 5A) compared with in normal adjacent tissue. Western blot analysis data further confirmed the increased expression of CAV1 protein expression in tumor tissue (Fig. 5B) compared with in normal adjacent tissue. These results indicated that the expression of CAV1 was elevated in breast tumor tissue.

Downregulation of CAV1 inhibits breast tumor cell proliferation and induces cell apoptosis and cell cycle arrest. To investigate the role the CAV1 in the regulation of tumor cell proliferation, downregulation of CAV1 was introduced in the breast tumor cells line MCF-7 and MDA-MB-231 cells by infecting cell with siRNA-CAV1 and siRNA-control. CAV1 expression was significantly reduced following siRNA-CAV1 infection (Fig. 6A and B) compared with the siRNA-control group. The colony formation experiment demonstrated that tumor cell growth is inhibited by downregulation of CAV1 (Fig. 6C) compared with the siRNA-control group. Additionally, the colony formation rate in the siRNA-CAV1 group was only $30 \%$ of the siRNA-control group (Fig. 6D). Cell viability was also decreased in the siRNA-CAV1 infected MCF-7 and MDA-MB-231 cells (Fig. 6E and F) compared with the siRNA-control group. Since cell proliferation was inhibited by the CAV1 expression, the effect of CAV1 on tumor cell apoptosis was evaluated. The flow cytometry analysis results indicated that downregulation of CAV1 with siRNA-CAV1 increased tumor cell apoptosis, as demonstrated by staining the infected cells with Annexin V and PI (Fig. 6G). The apoptotic cell ratio increased $\sim 2$-folds in the CAV1 downregulation cells, compared with the siRNA-control infected cells (Fig. 6H) in MCF-7 and MDA-MB-231 cells. Flow cytometry analysis also demonstrated that downregulation of CAV1 significantly increased the number of cells in the G0/G1 phase and decreased the 
A

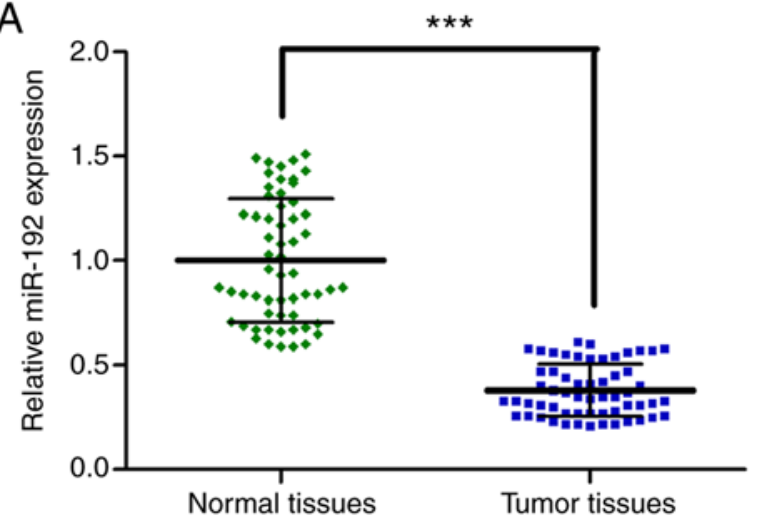

B

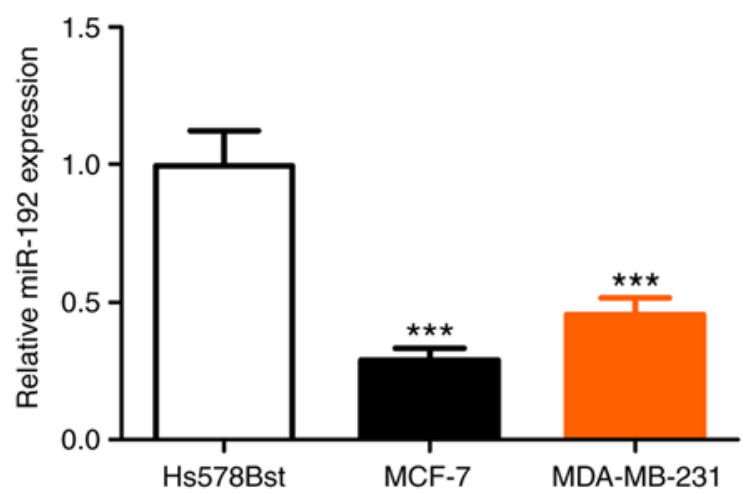

Figure 1. The expression of miR-192 in breast cancer tissues and cell lines. (A) The relative expression of miR-192 (normalized to U6) was detected and analyzed with reverse transcription-quantitative polymerase chain reaction in breast cancer and matched adjacent normal tissues. (B) Relative expression of miR-192 in normal Hs578Bst cells, and breast cancer MCF-7 and MDA-MB-231 cells. miR, microRNA. ${ }^{* * *} \mathrm{P}<0.001$.
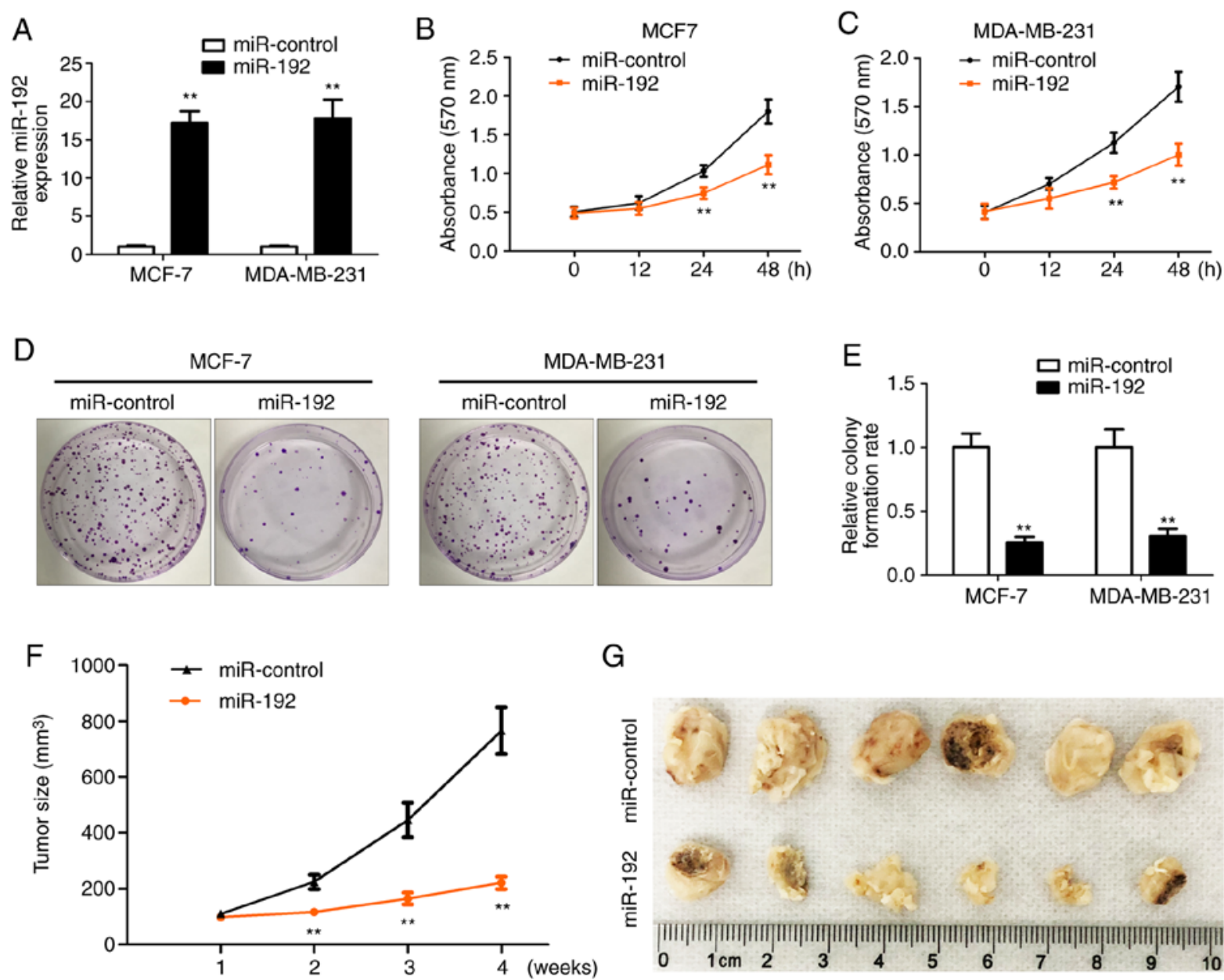

Figure 2. Overexpression of miR-192 inhibits the proliferation of breast cancer cells. (A) Reverse transcription-quantitative polymerase chain reaction was used to detect the transfection efficiency of miR-192 mimics in MCF-7 and MDA-MB-231 cells. (B and C) MTT assay was performed to detect cell survival. (D) Effect of upregulation of miR-192 on the long-term cell growth as determined by colony formation assay. (E) Colony number was quantified. (F) Breast tumor growth was monitored and measured every 7 days. (G) Tumor volume was calculated and analyzed. miR-192 mimic administration significantly inhibited tumor growth, compared with the control group. miR, microRNA. ${ }^{* *} \mathrm{P}<0.01$.

number of cells in the $\mathrm{S}$ phase, while there was no significant change in the G2/M phase (Fig. 6I and J). Collectively, these results demonstrated that downregulation of CAV1 inhibits breast tumor cell proliferation, and induces cell apoptosis and cell cycle arrest.

\section{Discussion}

The abnormal expression of miRNAs serves an important role in the cancer development and progression $(22,23)$. Numerous miRNAs, including miR-192, serve as tumor suppressors and 
A

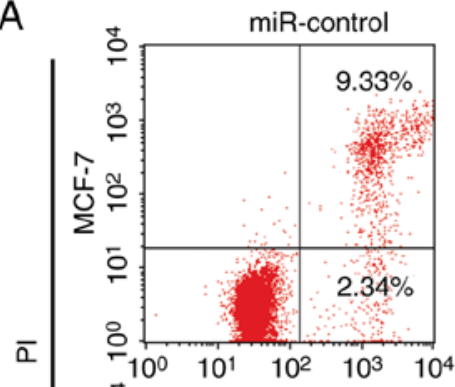

Annexin V-FITC
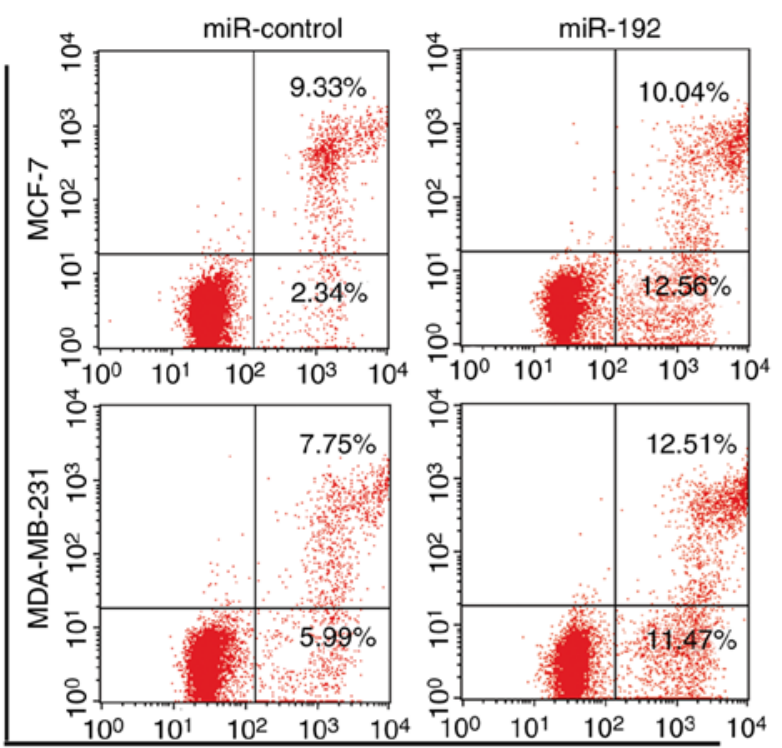

일
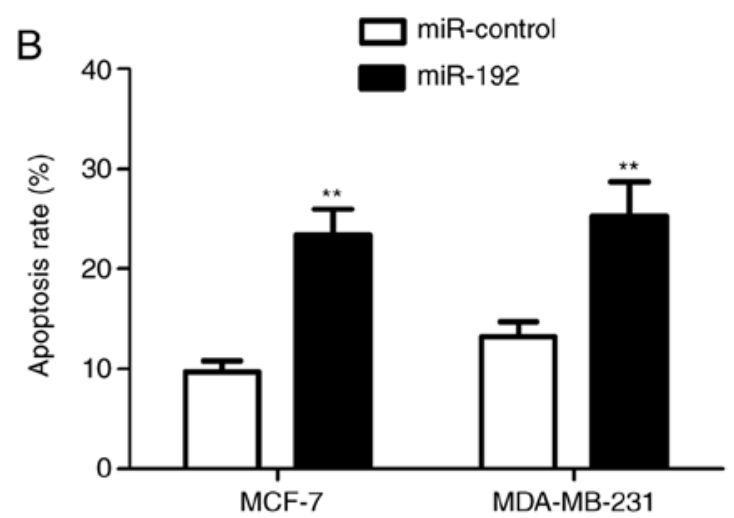

D 1007 miR-control

C

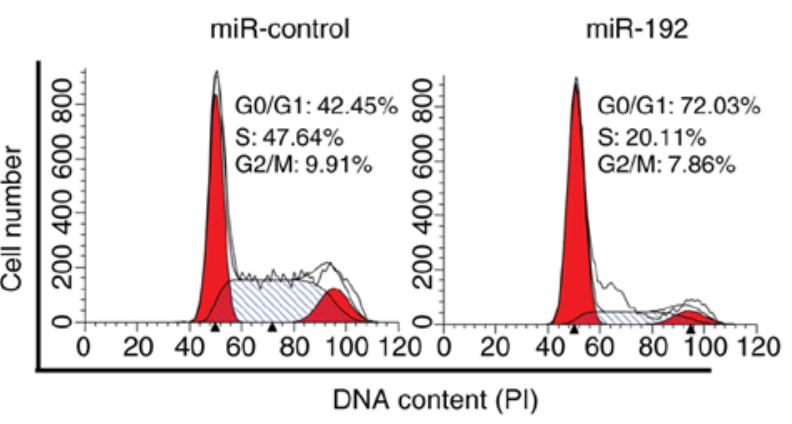

MCF-7

$E$

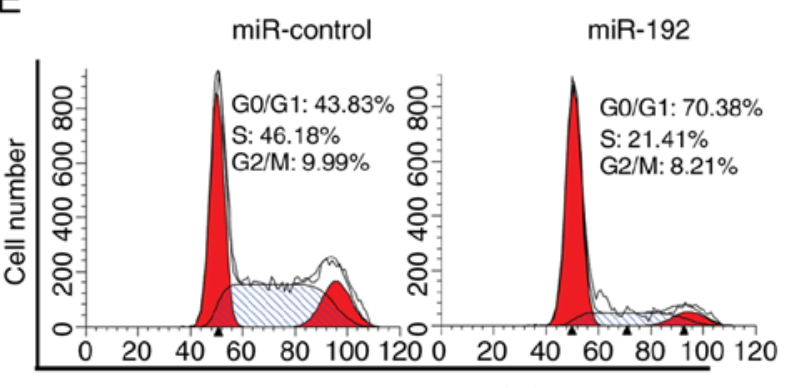

DNA content (PI)

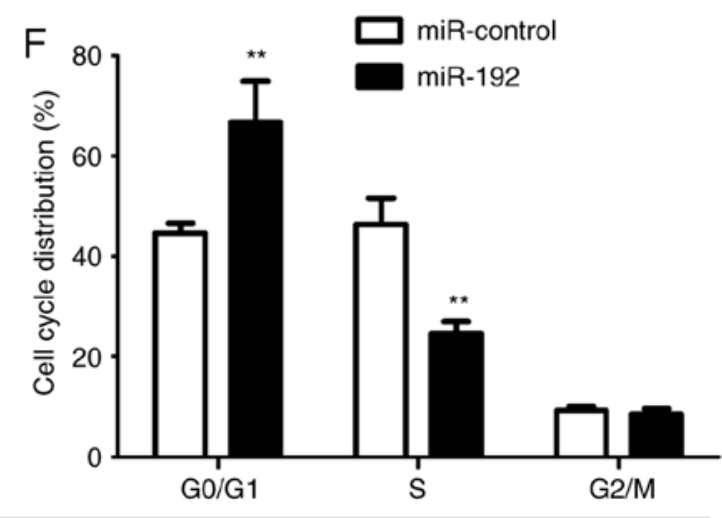

MDA-MB-231

Figure 3. miR-192 induces tumor cell apoptosis and cell cycle arrest. Tumor cell apoptosis was detected by Annexin-V FITC/PI double-staining in (A) MCF-7 and (B) MDA-MB-231 cells transfected with miR-192 mimics or miR-control, and apoptotic cells were defined as Annexin V-positive/PI-negative. (C-F) FACS analysis was used to detect the effect of miR-192 on the cell cycle in MCF-7 and MDA-MB-231 cells. FITC, fluorescein isothiocyanate; PI, propidium iodide; miR, microRNA. ${ }^{* *} \mathrm{P}<0.01$.

their aberrant expression serves critical roles in numerous human cancer types, including breast cancer, prostate cancer and colon cancer $(20,24)$. In 2003, miR-192 was confirmed by Lim et al (14). It is demonstrated to serve various roles in different human cancer types, such as gastric cancer, prostate cancer and neuroblastoma $(17,25,26)$. It has been demonstrated to be overexpressed in gastric cancer, hepatocellular carcinoma and neuroblastoma, while downregulated in colorectal cancer and hematological disorders, as well as in lymphoblastic leukemia (16-20). However, the expression of miR-192 and the role of miR-192 in breast cancer remain elusive. Breast cancer, which is the most commonly diagnosed cancer type in females, is still considered the secondary leading cause of cancer mortalities among females worldwide (1). Thus, it is 

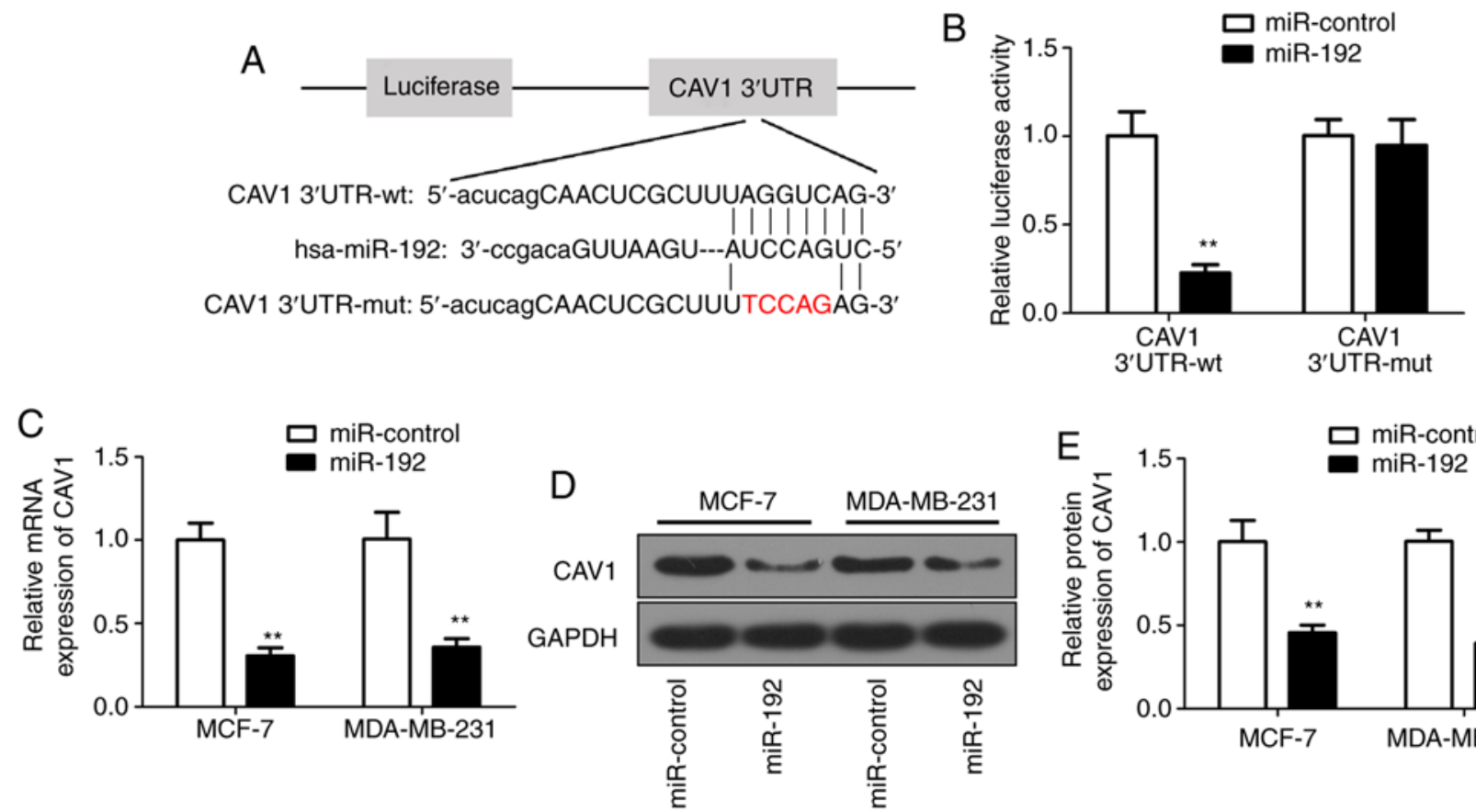

Figure 4. miR-192 targets CAV1 to repress its expression. (A) Bioinformatic analysis prediction indicated that miR-192 may target CAV1. (B) The effect of miR-192 on the luciferase intensity of pGL3/Luciferase-CAV1-3'UTR reporter was detected in 293T cells transfected with miR-192 mimics. (C-E) Reverse transcription-quantitative polymerase chain reaction and western blot analysis were performed to determine the effect of miR-192 on CAV1 mRNA in MCF-7 and MDA-MB-231 cells. CAV1, caveolin 1; UTR, untranslated region; miR, microRNA. ${ }^{* *} \mathrm{P}<0.01$.

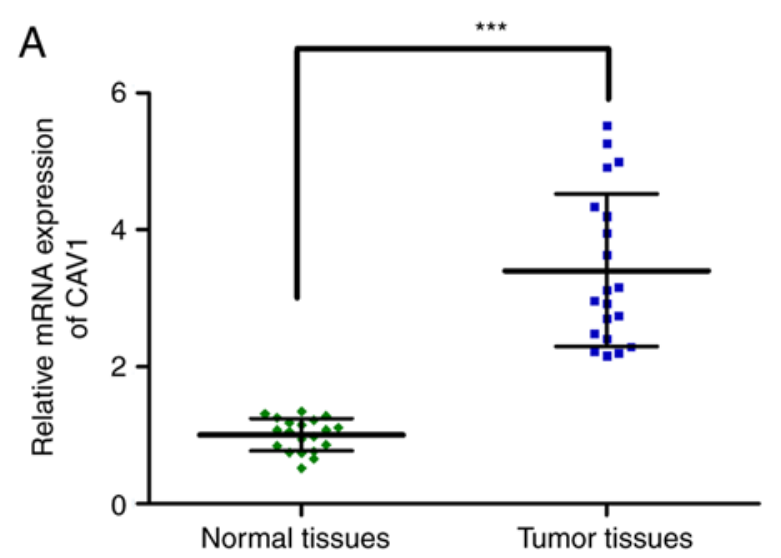

B

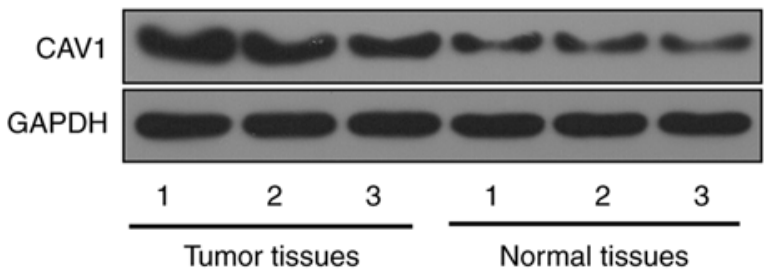

Figure 5. CAV1 expression in breast cancer tissues. (A) Reverse transcription-quantitative polymerase chain reaction was used to detect the expression of CAV1 RNA in breast cancer tissues and adjacent normal tissues. (B) Western blot analysis was used to detect the expression of CAV1 protein in 3 pairs of breast cancer tissues and normal tissue. CAV1, caveolin $1 .{ }^{* * *} \mathrm{P}<0.001$.

necessary to elucidate the role of miR-192 in the development of breast cancer. In the present study, the results indicated that miR-192 expression is significantly decreased in breast tumor tissue. This observation is accordance with other studies, which reported that miR-192 serves a tumor suppressor role and is downregulated in different tumor types (25-27). Further results demonstrated that overexpression of miR-192 in breast tumor cells significantly inhibited tumor cell proliferation and colony formation. Furthermore, overexpression of miR-192 induced tumor cell apoptosis and cell cycle arrest in the G0/G1 phase. Collectively, these results indicate that miR-192 may serve as a tumor suppressor in breast cancer.

Bioinformatic analysis predicted that miR-192 may target CAV1. CAV1, a $21 \mathrm{KDa}$ protein encoded by the CAV1 gene, is ubiquitously expressed in all cell types (28). In the past two decades, researchers focused on investigating the role of CAV1 in the tumor development determined that CAV1 is overexpressed in liver, colon, breast, kidney, lung and other cancer types (29). CAV1 has been reported to serve opposite roles as a tumor promotor or tumor suppressor, dependent on the cancer type and stage $(28,30)$. Previous studies reported that high expression of CAV1 induces tumorigenesis by inhibition of apoptosis, facilitation of anchorage-independent growth, antitumor drug resistance and promotion of tumor metastasis (30-32). Regarding its tumor suppressor role, overexpression of CAV1 inhibits tumor cell progression and prolonged survival rate in hepatocellular carcinoma $(33,34)$. Thus, investigation of the expression and regulation of CAV1 

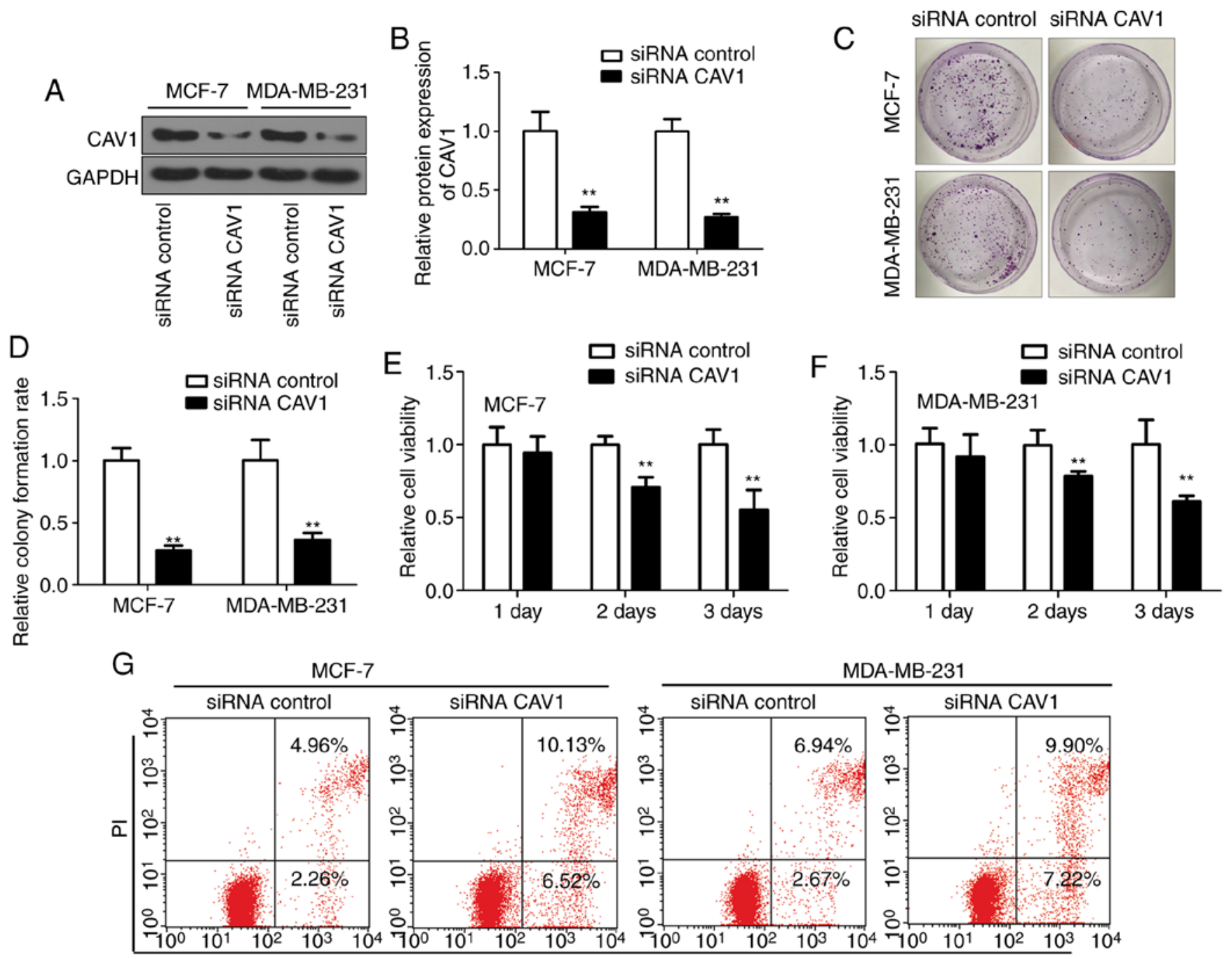

Annexin V-FITC
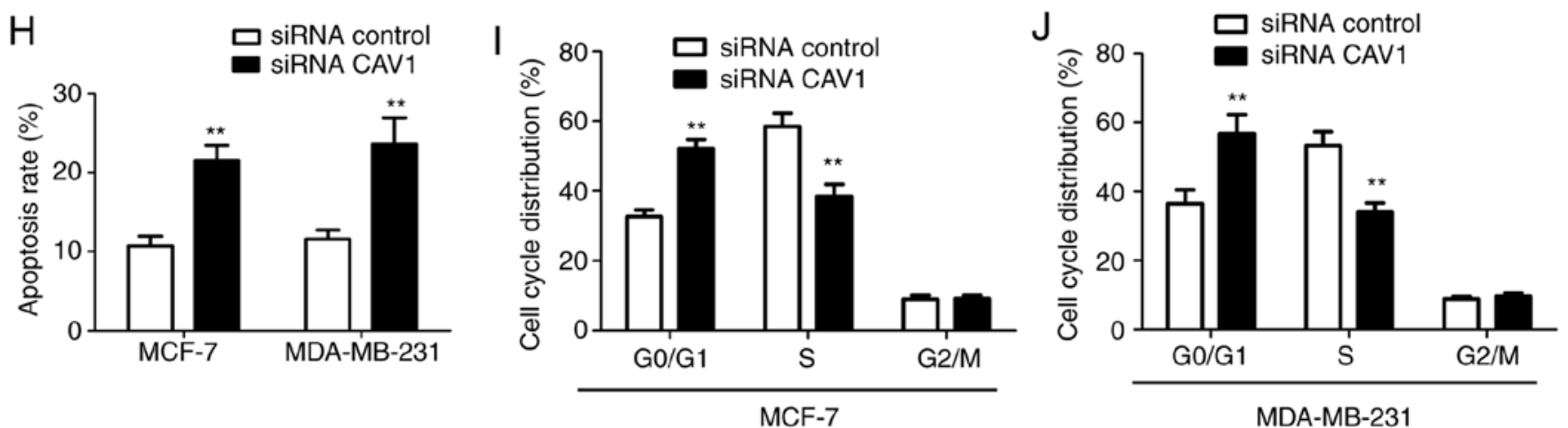

Figure 6. Downregulation of CAV1 inhibits the cell proliferation and induces the cell apoptosis and cell cycle arrest in breast cancer cells. The downregulation efficiency CAV1 by transfection of siRNA CAV1 in (A) MCF-7 and (B) MDA-MB-231 cells was determined by western blot analysis. (C and D) Colony formation assay was used to define the role of CAV1 on the long-term cell growth. (E and F) MTT assay detection of cell survival. (G and H) The effect of downregulation of CAV1 by transfection of CAV siRNA on cell apoptosis was detected by using Annexin V-FITC/PI double staining method in MCF-7 and MDA-MB-231 cells by flow cytometry. (I and J) FACS assay was used to detect the effect of siRNA CAV1 on the cell cycle in MCF-7 and MDA-MB-231 cells. CAV1, caveolin 1; siRNA, small interfering RNA; FITC, fluorescein isothiocyanate; PI, propidium iodide. ${ }^{* *} \mathrm{P}<0.01$.

by miRNA in breast tumor and breast tumor cells will further enhance the understanding of the role of CAV1 in breast cancer.

In the present study, it was demonstrated that miR-192 directly targets CAV1. CAV1 expression in breast tumor tissue and breast protein expression is negatively regulated by miR-192. Overexpression of miR-192 significantly decreased
CAV1 expression in breast tumor cells. This result indicated that miR-192 negatively regulates the expression of CAV1. Furthermore, the present data also demonstrated that CAV1 expression is increased in breast tumor tissues, compared with adjacent normal tissues. Downregulation of CAV1 in tumor cell inhibited tumor cell proliferation, and induced tumor cell 
apoptosis and cell cycle arrest. Therefore, these results demonstrated that miR-192 serves an important role in the regulation of breast tumor cell proliferation and apoptosis. miR-192 directly targeted CAV1, which was highly expressed in breast tumor tissues and tumor cells, to inhibit tumor growth and progression. Thus, the miR-192/CAV1 axis may have a potential as a therapeutic target for the treatment of breast cancer.

miR-192 expression was decreased in breast tumor tissues. Additionally, overexpression of miR-192 inhibited breast tumor cell proliferation, and induced tumor cell apoptosis and cell cycle arrest in the G0/G1 phase. Furthermore, miR-192 directly targeted CAV1 and negatively regulated the expression of CAV1 in breast tumor cells. CAV1 was highly expressed in breast tumor and downregulation of CAV1 inhibited tumor cell proliferation and induces tumor cell apoptosis and cell cycle arrest. Thus, the miR-192-CAV1 axis should be investigated as a potential target for treatment of breast cancer.

\section{Acknowledgements}

Not applicable.

\section{Funding}

No funding was received.

\section{Availability of data and materials}

The datasets used and/or analyzed during the current study are available from the corresponding author on reasonable request.

\section{Authors' contributions}

PC, YF and XL were involved in the study design, investigation, analysis and manuscript preparation. HZ, XS, BL, WJ, $\mathrm{XY}$ and NZ were involved in the investigation and analysis. All authors read and approved the final manuscript.

\section{Ethics approval and consent to participate}

The present human and animal studies were approved by the Ethics Committee of The Affiliated Luoyang Central Hospital of Zhengzhou University, and patients and healthy volunteers provided written informed consent. The research was carried out in accordance with the World Medical Association Declaration of Helsinki.

\section{Patient consent for publication}

All patients and healthy volunteers provided written informed consent prior to their inclusion within the study.

\section{Competing interests}

The authors declare that they have no competing interests.

\section{References}

1. Siegel RL, Miller KD and Jemal A: Cancer statistics, 2015. CA Cancer J Clin 65: 5-29, 2015.
2. Youlden DR, Cramb SM, Dunn NA, Muller JM, Pyke CM and Baade PD: The descriptive epidemiology of female breast cancer: An international comparison of screening, incidence, survival and mortality. Cancer Epidemiol 36: 237-248, 2012.

3. Cancer Genome Atlas Network: Comprehensive molecular portraits of human breast tumours. Nature 490: 61-70, 2012.

4. Bartel DP: MicroRNAs: Genomics, biogenesis, mechanism, and function. Cell 116: 281-297, 2004.

5. Bartel DP: MicroRNAs: Target recognition and regulatory functions. Cell 136: 215-233, 2009.

6. Ruvkun G: Clarifications on miRNA and cancer. Science 311: 36-37, 2006.

7. Xi Y, Shalgi R, Fodstad O, Pilpel Y and Ju J: Differentially regulated micro-RNAs and actively translated messenger RNA transcripts by tumor suppressor p 53 in colon cancer. Clin Cancer Res 12: 2014-2024, 2006.

8. Zhang B, Pan X, Cobb GP and Anderson TA: microRNAs as oncogenes and tumor suppressors. Dev Biol 302: 1-12, 2007.

9. Kurozumi S, Yamaguchi Y, Kurosumi M, Ohira M, Matsumoto H and Horiguchi J: Recent trends in microRNA research into breast cancer with particular focus on the associations between microRNAs and intrinsic subtypes. J Hum Genet 62: 15-24, 2017.

10. Kayani M, Kayani MA, Malik FA and Faryal R: Role of miRNAs in breast cancer. Asian Pac J Cancer Prev 12: 3175-3180, 2011.

11. Mulrane L, McGee SF, Gallagher WM and O'Connor DP: miRNA dysregulation in breast cancer. Cancer Res 73: 6554-6562, 2013.

12. Serpico D, Molino L and Di Cosimo S: microRNAs in breast cancer development and treatment. Cancer Treat Rev 40: 595-604, 2014.

13. Takahashi Ru, Miyazaki H and Ochiya T: The roles of microRNAs in breast cancer. Cancers (Basel) 7: 598-616, 2015.

14. Lim LP, Lim LP, Glasner ME, Yekta S, Burge CB and Bartel DP: Vertebrate microRNA genes. Science 299: 1540, 2003.

15. Pichiorri F, Suh SS, Rocci A, De Luca L, Taccioli C, Santhanam R, Zhou W, Benson DM Jr, Hofmainster C, Alder H, et al: Downregulation of p53-inducible microRNAs 192, 194, and 215 impairs the p53/MDM2 autoregulatory loop in multiple myeloma development. Cancer Cell 18: 367-381, 2010.

16. Schotte D, De Menezes RX, Akbari Moqadam F, Khankahdani LM, Lange-Turenhout E, Chen C, Pieters R and Den Boer ML: MicroRNA characterize genetic diversity and drug resistance in pediatric acute lymphoblastic leukemia. Haematologica 96: 703-711, 2011.

17. Feinberg-Gorenshtein G, Guedj A, Shichrur K, Jeison M, Luria D, Kodman Y, Ash S, Feinmesser M, Edry L, Shomron N, et al: MiR-192 directly binds and regulates Dicerl expression in neuroblastoma. PLoS One 8: e78713, 2013.

18. Song B, Wang Y, Kudo K, Gavin EJ, Xi Y and Ju J: miR-192 Regulates dihydrofolate reductase and cellular proliferation through the p53-microRNA circuit. Clin Cancer Res 14: 8080-8068, 2008.

19. Tan Y, Ge G, Pan T, Wen D, Chen L, Yu X, Zhou X and Gan J: A serum microRNA panel as potential biomarkers for hepatocellular carcinoma related with hepatitis B virus. PLoS One 9: e107986, 2014.

20. Jin Z, Selaru FM, Cheng Y, Kan T, Agarwal R, Mori Y, Olaru AV, Yang J, David S, Hamilton JP, et al: MicroRNA-192 and-215 are upregulated in human gastric cancer in vivo and suppress ALCAM expression in vitro. Oncogene 30: 1577-1585, 2011.

21. Livak KJ and Schmittgen TD: Analysis of relative gene expression data using real-time quantitative PCR and the 2(-Delta Delta C(T)) method. Methods 25: 402-408, 2001.

22. Augello C, Vaira V, Caruso L, Destro A, Maggioni M, Park YN, Montorsi M, Santambrogio R, Roncalli M and Bosari S: MicroRNA profiling of hepatocarcinogenesis identifies C19MC cluster as a novel prognostic biomarker in hepatocellular carcinoma. Liver Int 32: 772-782, 2012.

23. Goto Y, Kojima S, Nishikawa R, Enokida H, Chiyomaru T, Kinoshita T, Nakagawa M, Naya Y, Ichikawa T and Seki N: The microRNA-23b/27b/24-1 cluster is a disease progression marker and tumor suppressor in prostate cancer. Oncotarget 5: 7748-7759, 2014.

24. Jin Y, Lu J, Wen J, Shen Y and Wen X: Regulation of growth of human bladder cancer by miR-192. Tumor Biol 36: 3791-3797, 2015.

25. Xu YJ and Fan Y: MiR-215/192 participates in gastric cancer progression. Clin Transl Oncol 17: 34-40, 2015.

26. Sun J, Fan Z, Lu S, Yang J, Hao T and Huo Q: MiR-192 suppresses the tumorigenicity of prostate cancer cells by targeting and inhibiting nin one binding protein. Int J Mol Med 37: 485-492, 2016. 
27. Feng S, Cong S, Zhang X, Bao X, Wang W, Li H, Wang Z, Wang G, Xu J, Du B, et al: MicroRNA-192 targeting retinoblastoma 1 inhibits cell proliferation and induces cell apoptosis in lung cancer cells. Nucleic Acids Res 39: 6669-6678, 2011.

28. Gupta R, Toufaily C and Annabi B: Caveolin and cavin family members: Dual roles in cancer. Biochimie 107: 188-202, 2014.

29. Burgermeister E, Liscovitch M, Röcken C, Schmid RM and Ebert MP: Caveats of caveolin-1 in cancer progression. Cancer Lett 268: 187-201, 2008.

30. Wang Z, Wang N, Liu P, Peng F, Tang H, Chen Q, Xu R, Dai Y, Lin Y, Xie X, et al: Caveolin-1, a stress-related oncotarget, in drug resistance. Oncotarget 6: 37135-37150, 2015.

31. Patani N, Martin LA, Reis-Filho JS and Dowsett M: The role of caveolin-1 in human breast cancer. Breast Cancer Res Treat 131: $1-15,2012$.

32. Savage K, Lambros MB, Robertson D, Jones RL, Jones C, Mackay A, James M, Hornick JL, Pereira EM, Milanezi F, et al: Caveolin 1 is overexpressed and amplified in a subset of basal-like and metaplastic breast carcinomas: A morphologic, ultrastructural, immunohistochemical, and in situ hybridization analysis. Clin Cancer Res 13: 90-101, 2007.
33. Yang SF, Yang JY, Huang CH, Wang SN, Lu CP, Tsai CJ, Chai CY and Yeh YT: Increased caveolin-1 expression associated with prolonged overall survival rate in hepatocellular carcinoma. Pathology 42: 438-445, 2010.

34. Chatterjee M, Ben-Josef E, Thomas DG, Morgan MA, Zalupski MM, Khan G, Andrew Robinson C, Griffith KA, Chen CS, Ludwig T, et al: Caveolin-1 is associated with tumor progression and confers a multi-modality resistance phenotype in pancreatic cancer. Sci Rep 5: 10867, 2015.

(1)(9) This work is licensed under a Creative Commons Attribution-NonCommercial-NoDerivatives 4.0 International (CC BY-NC-ND 4.0) License. 